\title{
Exploration of the Danggui Buxue Decoction Mechanism Regulating the Balance of ESR and AR in the TP53-AKT Signaling Pathway in the Prevention and Treatment of POF
}

\author{
Huaiquan Liu $\mathbb{D},{ }^{1,2}$ Hong Yang, ${ }^{3}$ Zhong Qin, ${ }^{2}$ Yunzhi Chen, ${ }^{2}$ Haiyang Yu $\mathbb{D},{ }^{2}$ Wen Li, ${ }^{2}$ \\ Xing Zhu, ${ }^{2}$ Jingwen Cai, ${ }^{3}$ Jing Chen $\mathbb{D}^{4},{ }^{4}$ and Mengzhi Zhang $\mathbb{D}^{5}$ \\ ${ }^{1}$ Shandong Xiandai University, Jinan, Shandong 250104, China \\ ${ }^{2}$ Guizhou University of Traditional Chinese Medicine, Guiyang, Guizhou 550025, China \\ ${ }^{3}$ Shanghai Shuguang Hospital Affiliated with Shanghai University of Traditional Chinese Medicine, Shanghai 201203, China \\ ${ }^{4}$ Shanghai Municipal Hospital of Traditional Chinese Medicine, Shanghai University of Traditional Chinese Medicine, \\ Shanghai 200071, China \\ ${ }^{5}$ The Second Affiliated Hospital of Nanchang University, Nanchang, Jiangxi 330006, China
}

Correspondence should be addressed to Jing Chen; cj1275@126.com and Mengzhi Zhang; ndefy20385@ncu.edu.cn

Received 24 May 2021; Accepted 16 November 2021; Published 30 December 2021

Academic Editor: Barbara Romano

Copyright (C) 2021 Huaiquan Liu et al. This is an open access article distributed under the Creative Commons Attribution License, which permits unrestricted use, distribution, and reproduction in any medium, provided the original work is properly cited.

\begin{abstract}
Objective. The purpose of this study was to explore the molecular mechanism of Danggui Buxue Decoction (DBD) intervening premature ovarian failure (POF). Methods. The active compounds-targets network, active compounds-POF-targets network, and protein-protein interaction (PPI) network were constructed by a network pharmacology approach: Gene Ontology (GO) function and Kyoto Encyclopedia of Gene and Genome (KEGG) pathway analysis by DAVID 6.8 database. The molecular docking method was used to verify the interaction between core components of DBD and targets. Then, High-Performance Liquid Chromatography (HPLC) analysis was used to determine whether the DBD contained two key components including quercetin and kaempferol. Finally, the estrous cycle, organ index, ELISA, and western blot were used to verify that mechanism of DBD improved POF induced by cyclophosphamide (CTX) in rats. Results. Based on the network database including TCMSP, Swiss Target Prediction, DisGeNET, DrugBank, OMIM, and Malacard, we built the active compounds-targets network and active compounds-POF-targets network. We found that 2 core compounds (quercetin and kaempferol) and 5 critical targets (TP53, IL6, ESR1, AKT1, and AR) play an important role in the treatment of POF with DBD. The GO and KEGG enrichment analysis showed that the common targets involved a variety of signaling pathways, including the reactive oxygen species metabolic process, release of Cytochrome $\mathrm{C}$ from mitochondria and apoptotic signaling pathway, p53 signaling pathway, the PI3K-Akt signaling pathway, and the estrogen signaling pathway. The molecular docking showed that quercetin, kaempferol, and 5 critical targets had good results regarding the binding energy. Chromatography showed that $\mathrm{DBD}$ contained quercetin and kaempferol compounds, which was consistent with the database prediction results. Based on the above results, we found that the process of DBD interfering POF is closely related to the balance of ESR and AR in TP53-AKT signaling pathway and verified animal experiments. In animal experiments, we have shown that DBD and its active compounds can effectively improve estrus cycle of POF rats, inhibit serum levels of FSH and LH, protein expression levels of Cytochrome C, BAX, p53, and IL6, and promote ovary index, uterine index, serum levels of $\mathrm{E}_{2}$ and $\mathrm{AMH}$, and protein expression levels of AKT1, ESR1, AR, and BCL2. Conclusions. DBD and its active components could treat POF by regulating the balance of ESR and AR in TP53-AKT signaling pathway.
\end{abstract}

\section{Introduction}

Premature ovarian failure (POF) is a disorder of the female reproductive system under the age of 40 years due to reduced ovarian function for various reasons. Generally, clinical manifestations include hot flashes, night sweats, hypaphrodisia, infertility, and persistent amenorrhea for more than 6 months [1]. There are several biochemical measurements that involve estradiol $\left(\mathrm{E}_{2}\right)$, antimullerian hormone $(\mathrm{AMH})$, follicular stimulating hormone (FSH), and luteinizing 
hormone (LH) to predict reproductive potential [2]. The global incidence of POF is approximately $1 \%$, which is closely related to autoimmune diseases, neurological disorders, iatrogenic injuries, unhealthy living habits, etc. [3]. Currently, hormone replacement therapy (HRT) is mainly used for the treatment of POF. However, long-term treatment may easily cause anxiety, depression, irritability, and other adverse reactions. More seriously, it may even induce certain complications such as endometrial cancer, breast cancer, and unexplained vaginal bleeding [4]. What is noteworthy is that the traditional Chinese medicine (TCM) as complementary medicine therapy has not only achieved extremely effective results, but also greatly reduced the incidence of adverse reactions and complications [5].

Chinese herbal medicines and their derived secondary metabolism have different biological characteristics, which can prevent and treat a variety of human diseases [6], such as enhancing immunity, anti-inflammatory [7, 8], antiviral [9], regulation of oxidative stress [10], antianxiety [11], anticancer [12], and prevention of the genitourinary systems diseases [13]. It is worth noting that network pharmacology is an emerging branch of pharmacology composed of computer science, biology, medicine, and bioinformatics. It is usually committed to establishing network prediction models through public databases and high throughput to initially predict the molecular mechanism of drug treatment. Molecular docking is an important method for drug development and is used to study the interaction between receptor proteins and drugs. Therefore, the preliminary screening of targets and signaling pathways through the docking of network pharmacology and molecules and then using animal experiments for verification provide a promising way to quickly explore the pharmacological mechanism of traditional Chinese medicine.

1.1. Traditional Chinese Medicine. Gynecology believes that POF belongs to the categories of "blood depletion," "amenorrhea," and "infertility" and proposes that sufficient vital essence in the kidney is the basis for menstruation, and a deficient kidney essence would cause menstrual blood to dry up to form blood depletion. Miraculous Pivot indicates that women need sufficient blood to support themselves during menstruation and pregnancy; blood cannot fill the uterus and ovaries and other organs in the event of deficiency, resulting in amenorrhea and infertility. Danggui Buxue Decoction (DBD) is a classic recipe for nourishing "Qi" and enriching "Blood," among which Radix Astragali can nourish yin and enrich blood, Radix Angelicae sinensis can promote blood circulation and nourish uterus. The combination of these two herbs can function together to enrich blood and nourish uterus. Modern medical studies have found that DBD and its main chemical constituents have a good therapeutic effect on women's reproductive problems such as POF, polycystic ovary syndrome (PCOS), irregular menstruation, and menopause $[14,15]$. DBD has a series of pharmacological effects, including promoting hematopoiesis, increasing immunity, and regulating hormone levels [16]. $\mathrm{DBD}$ can increase the level of serum $\mathrm{E}_{2}$ and decrease the level of serum $\mathrm{FSH}$ and $\mathrm{LH}$ to improve hormone levels in ovariectomized (OVX) female rats and increase the weight of uterus $[17,18]$. The serum of the drug in DBD can accelerate ovarian granular cell proliferation in rats and inhibit the activity of reactive oxygen species (ROS) free radicals to achieve the purpose of interfering with POF by reducing the protein expression level of the Caspase-3 [19].

Additionally, the pathogenesis of POF is still unclear and it is urgent to find its potential therapeutic targets. Therefore, this study mainly combines network pharmacology and molecular docking technology to explore potential targets and active compounds for DBD treatment of POF. The experiments in rats further explore the intervention mechanism of DBD on POF, which can provide important reference for the treatment of POF with TCM.

\section{Materials and Methods}

\subsection{Network Pharmacology Analysis}

2.1.1. Screening of Active Compounds and Collection of Targets. The compounds were searched by inputting "Huangqi" and "Danggui" as keywords on Traditional Chinese Medicine Systems Pharmacology Database and Analysis Platform (TCMSP, https://tcmspw.com/tcmsp.php). Based on parameters of pharmacokinetics (ADME), oral bioavailability $(\mathrm{OB}) \geq 30 \%$ and drug-likeness $(\mathrm{DL}) \geq 0.18$ were selected as the conditions for screening active compounds to determine the active compounds in DBD and obtain corresponding sequence of Canonical SMILES after further comparisons [20]. "Related Targets" in the TCMSP database and "Canonical SMILES" (the top 15 target targets were screened based on correlation) in the Swiss Target Prediction database (https://www.Swisstargetprediction.ch) were utilized to jointly predict the targets of active compounds [21]. All targets were introduced by the Uniprot database (https:// www.Uniprot.org/), and then a network of "Active compound targets” was built with Cytoscape 3.7.2 software [22].

2.1.2. Construction of Active Compound-POF-Targets Network. POF related targets were searched by entering "Premature ovarian failure" as keyword in DisGeNET (https:// www.disgenet.org/), DrugBank (https://www.drugbank.ca), OMIM (https://omim.org/), and Malacard (https://www. malacards.org/) [23]. The Venn diagrams of the targets of $\mathrm{DBD}$ and POF were drawn out using $\mathrm{R}$, and then the screening of common targets as important potential targets intervening POF in DBD was performed. The network of "active compound-POF-targets" was built using Cytoscape 3.7.2 software.

\subsubsection{Construction of Protein-Protein Interaction (PPI)} Network. We introduced the targets intervening POF in DBD into STRING.11.0 (https://string-db.org/) platform where the mutual score was set as "medium confidence $=0.4$ " and the species was set as "Homo sapiens" in order to construct a PPI network [24]. The topological parameters of the PPI network were acquired by the "Network Analysis" function in the cytoscape3.7.2 software, among which the greater degree value is the most important in the 
PPI network. The top five core targets were selected based on degree scores as important targets for DBD to interfere with POF. Then, the PPI networks of the 5 core targets were constructed by the STRING.11.0 platform and setting "highest confidence $=0.9$ " and the species "Homo sapiens".

2.1.4. Biological Function and Pathway Analysis. The GO function and the analysis of the KEGG pathway were performed for POF targets that intervene in DBD and "Homo sapiens" species in the DAVID 6.8 database (https://david. ncifcrf.gov/home.jsp) [25]. The functions of the GO and KEGG pathways were subjected to visualization analysis using ggplot in the $\mathrm{R}$ package.

2.1.5. Analysis of Molecular Docking between Active Compounds and the Core Protein Receptor. The top five targets of the degree value in the PPI network were selected as protein receptors, and the active compounds of the top 2 degrees were selected as ligands of the active compounds in the DBD intervening in POF for verifying the molecular docking, respectively. The target protein files in PDB format and the active compound files in SDF were obtained from the RSCB PDB database (https://www.rcsb.org/) and the PubChem database. Chem Office software was used to perform mol2 format conversion and energy minimization in active compounds. After water molecules, original ligands, and polarized hydrogen from target proteins were removed via PyMOL software, the affinity and binding energy between the target protein and the active compounds were analyzed by Autodock Vina and Python scripts. Meanwhile, the binding energy (affinity) $\leq-$ $5.0 \mathrm{~kJ} / \mathrm{mol}$ was a screening condition in this study [26].

\subsection{Experimental Validation}

2.2.1. Drugs and Reagents. The Radix Angelicae sinensis granules (Cat:0119371) and Radix Astragali granules (Cat: 0099211) are both produced by Guangdong Yifang Pharmaceutical Co., Ltd. According to clinical application, the approximate dose of DBD is Radix Astragali $30 \mathrm{~g}$ and Radix Angelicae sinensis $6 \mathrm{~g}$, configured according to the ratio of Radix Astragali: Radix Angelicae sinensis $=5: 1$, and $2 \mathrm{~g}$ of Radix Astragali granules are equivalent to $10 \mathrm{~g}$ of Radix Astragali decoction pieces, and $3 \mathrm{~g}$ of Radix Angelicae sinensis granules are equivalent to $10 \mathrm{~g}$ of Radix Angelicae sinensis decoction pieces. We dissolved $6 \mathrm{~g}$ of Radix Astragali granules (equivalent to $30 \mathrm{~g}$ of Radix Astragali decoction pieces) and $1.8 \mathrm{~g}$ of Radix Angelicae sinensis granules (equivalent to $6 \mathrm{~g}$ of Radix Angelicae sinensis decoction pieces) each day in $50 \mathrm{ml}$ of water solution to prepare a DBD solution with a crude drug concentration of $0.72 \mathrm{~g} / \mathrm{ml}$.

We used the following drugs and reagents: CTX (Shanxi Pude Pharmaceutical Co., Ltd., No. H14023686), quercetin, kaempferol (Chengdu Pusi Biological Technology Co., Ltd., Nos. PS012093 and PS011676), E, AMH, FSH, LH (Wuhan Huamei Biological Engineering Co., Ltd., Nos. CSB-E05110r, CSB-E11162r, CSB-E06869r, and CSB-E12654r); GAPDH (Hangzhou Xianzhi Biological Co., Ltd., No. AB-P-R 001),
HRP-labeled goat anti-rabbit secondary antibody (Wuhan Boster Biological Engineering Co., Ltd., No. BA1054), rabbit monoclonal antibody AKT1(Cell signaling, No. \#75692), rabbit monoclonal antibody Cytochrome $\mathrm{C}$ (abcam, No. Ab133504), rabbit polyclonal antibodies ESR1, BCL2, BAX (Wuhan Sanying Biotechnology Co., Ltd., Nos. 21244-1-AP, 12789-1-AP, and 50599-2-lg), and rabbit polyclonal antibodies AR, p53, IL6 (Affinity, Nos. DF6783, AF0865, and DF6087).

2.2.2. Chromatographic Analysis. The mixture of Radix Astragali granule $6 \mathrm{~g}$ and Radix Angelicae sinensis granule $1.8 \mathrm{~g}$ was dissolved in $50 \mathrm{ml}$ ultra-pure water to make DBD. $1 \mathrm{mg}$ standard product of quercetin is dissolved in $1 \mathrm{ml}$ methanol. $1 \mathrm{mg}$ standard product of kaempferol is dissolved in $1 \mathrm{ml}$ methanol. Each sample was centrifuged at $4^{\circ} \mathrm{C}$ at $12000 \mathrm{rpm}$ for $10 \mathrm{~min}$. The instrument used in this experiment is the highperformance liquid chromatography, equipped with DAD detector. The chromatographic column was Diamonsil C18 (2) $(250 * 4.6 \mathrm{~mm}, 5 \mu \mathrm{m})$, the flow rate was $1 \mathrm{ml} / \mathrm{min}$, the column temperature was $35^{\circ} \mathrm{C}$, the detection wavelength was $360 \mathrm{~nm}$, the mobile phase was methanol:0.1\% phosphoric acid water/ $50: 50$, and the injection volume was $10 \mu \mathrm{l}$.

2.2.3. Animals. Fifty female Sprague Dawley (SD) rats of healthy specific pathogen-free (SPF) grade (8 weeks old, $190 \pm 20 \mathrm{~g})$ were purchased from Changsha Tianqin Biotechnology Co., Ltd. (animal license number: SCXK(Xiang) 2019-0014). Rats were housed at a room temperature of $21 \pm 2^{\circ} \mathrm{C}$, humidity of $55 \pm 5 \%$, and normal light circadian rhythm, given a standard diet and distilled water, allowed to eat freely, and subjected to adaptive feeding for 7 days. The study was approved by the Institutional Ethics Committee of The Second Affiliated Hospital of Nanchang University and the examination and approval no. review [2021], no. (A709).

2.2.4. Model and Administration. Fifty female SD rats were randomly divided into control group $(n=10)$, model group $(n=10)$, DBD group $(n=10)$, quercetin group $(n=10)$, and kaempferol group $(n=10)$. The POF model was induced in rats. First, $200 \mathrm{mg} / \mathrm{kg}$ CTX was injected intraperitoneally on the first day and then $8 \mathrm{mg} / \mathrm{kg}$ CTX for the following 14 days [27]. DBD (7.2 g/kg/d, crude drug concentration) was administered to rats in DBD group, quercetin $(15 \mathrm{mg} / \mathrm{kg} / \mathrm{d})$ [28] to rats in quercetin group, and kaempferol $(40 \mathrm{mg} / \mathrm{kg} / \mathrm{d})$ [29] to rats in kaempferol group for 4 weeks, while the rats in the control group and the rats in the model group received sterile distilled water by gavage $(10 \mathrm{ml} / \mathrm{kg} / \mathrm{d})$ [30]. After 4 weeks of drug intervention, the estrus cycle was observed for 2 weeks, and then all rats were sacrificed.

2.2.5. Evaluation of Estrous Cycles. Every day at 8:00 am, all rats were observed in the estrous cycle. Vaginal secretions were collected in $20 \mu \mathrm{l} 9 \% \mathrm{NaCl}$ solution and smeared on the glass slide. After natural drying, soak in hematoxylin dye solution for dyeing for 5-10 min; rinse in running water; perform color separation with hydrochloric acid and ethanol for a few seconds; soak in tap water for $5 \mathrm{~min}$; stain with 
eosin dye solution for $3 \mathrm{~min}$; rinse in running water; dry, covered with slides; and microscopically inspect.

2.2.6. Organ Index. After sacrifice, both the ovaries and uterine were removed surgically and weighed. The ovary index and uterine index were calculated: ovary index $=$ the wet weight of bilateral ovaries $(g)$ /body weight $(g) \times 100 \%$, uterine index $=$ the wet weight of bilateral uterine $(g) /$ body weight $(g) \times 100 \%$.

2.2.7. ELISA. Optical densities (OD) were read at $450 \mathrm{~nm}$ and concentrations of $\mathrm{E}_{2}, \mathrm{AMH}, \mathrm{FSH}$, and $\mathrm{LH}$ were determined by comparison with standard curves.

2.2.8. Western Blot. Ovary tissues were fully split by proteinlysis buffer. Then, supernatant was obtained after centrifugation. The sample protein was $50 \mu \mathrm{g}$. Then, we performed gel preparation, electrophoresis, membrane transfer, and then blocking in 5\% skim milk for $2 \mathrm{~h}$. The membranes were incubated at $4^{\circ} \mathrm{C}$ overnight with antiGAPDH (1:2000), AKT1 (1:1000), BAX (1:5000), Cytochrome C (1: 5000), ESR1 (1:1000), IL6 (1:1000), p53 (1: 1000), AR (1:1000), and BCL2 $(1: 2000)$. The next day, we wash the membranes, second antibody incubation at $37^{\circ} \mathrm{C}$ for 2 hours. The enhanced chemiluminescence (ECL) substrate developed in membranes. The result of the image was analyzed using ImageJ software.

2.2.9. Data Analysis. All statistical analyses were executed with the SPSS 24.0 software program. Normal distribution was presented as mean \pm standard deviation (SD). When the variance is homogeneous, LSD is used for pairwise comparison between groups. The comparison of results between multiple groups adopts one-way analysis of variance. When the data is abnormal distribution, the distributions were presented as median and interquartile ranges. The data were assessed with the nonparametric Kruskal-Wallis test, and the Nemenyi method was used to compare the two groups. All results were considered statistically significant with $P<0.05$.

\section{Results}

3.1. Active Compounds and Targets of DBD. 22 active compounds in DBD were selected from the TCMSP database according to $\mathrm{OB} \geq 30 \%$ and $\mathrm{DL} \geq 0.18$. After comparing with the PubChem database, MOL000398-isoflavanone was deleted because it does not have matching compounds and Canonical SMILES sequences of the remaining 21 active compounds were obtained. The target compounds in DBD were searched from TCMSP and Swiss Target Prediction databases where 183 and 155 corresponding targets were obtained, respectively, after identifying their names in combination with Uniprot database. 312 targets remained after deleting duplicates. Construction and analysis of the relationship between the active compound and the target in DBD were carried out by Cytoscape 3.7.2 software, and then we obtained the "active compound-target" network diagram, as shown in Figure 1. There are 333 nodes (312 targets, 21 active compounds) and 678 edges in the figure where circles in light blue represent the targets, pink represents the active compounds of Radix Astragali, and orange represents the active compounds of Radix Angelicae sinensis.

3.2. Potential Targets of DBD-Intermediating POF. 160, 101, 99, and 366 known targets related to POF were searched from DisGeNET, DrugBank, OMIM, and Malacard databases, respectively. After deleting the duplicates, 592 POF-related pathogenic targets were obtained. Through the Venn diagram, the intersections of the active compound and the POF-related pathogenic 36 targets were obtained, as shown in Figure 2.

3.3. Active Compounds-POF-Targets Network. Related targets of intervening POF in DBD as well as the corresponding active compounds were inputted into Cytoscape 3.7.2 software to build "active compounds-POF-targets" network diagram, as shown in Figure 3. In the figure, there are 55 nodes (18 active compounds, 36 targets, and POF) and 143 edges, circles in light green represent the targets, $\mathrm{V}$ shapes represent the active compounds (green represents Radix Astragali, and orange represents Radix Angelicae sinensis), and the hexagon represents POF. After analysis of the mapping using the Network Analysis plug-in, it was found that the top 2 core active compounds with the degree value from high to low were MOL000098-quercetin and MOL000422-kaempferol. The topological parameters of the active core compounds mentioned above in the network of "active compounds-POF targets" are shown in Table 1.

3.4. PPI Network. The protein -protein interaction (PPI) network was obtained from the STRING.11.0 database and redrawn by Cytoscape 3.7.2, as shown in Figure 4(a). There are 36 nodes (target protein) and 228 edges (protein interaction) in the PPI network diagram. The size and depth of the node color of the node represent the scale of degree value. The results of network topology analysis showed that five targets selected according to degree value were TP53, IL6, ESR1, AKT1, and AR, as shown in Table 2. Then we set "highest confidence $=0.9$ " in the STRING.11.0 database to build a PPI network of five core targets, as shown in Figure 4(b).

3.5. Biological Function and Pathway. The results of the analysis of the GO function showed that 919 enrichment results were obtained after screening $(P<0.05)$, including 829 biological processes (BP), 8 cell compositions (CC), and 82 molecular functions (MF). In the study, the top $20 \mathrm{BP}$, $\mathrm{MF}$, and $8 \mathrm{CC}$ results were screened as per $P$-value for mapping a histogram, as shown in Figure 5, where BP mainly covered reactive oxygen species metabolic process, release of Cytochrome $\mathrm{C}$ from mitochondria, and apoptotic signaling pathway, CC mainly included endoplasmic reticulum and mitochondrion, and MF generally were related to aromatase activity and steroid binding.

KEGG analysis showed that 41 signaling pathways $(P<0.05)$ were involved in the p53 signaling pathway, PI3K-Akt signaling pathway, estrogen signaling pathway, 


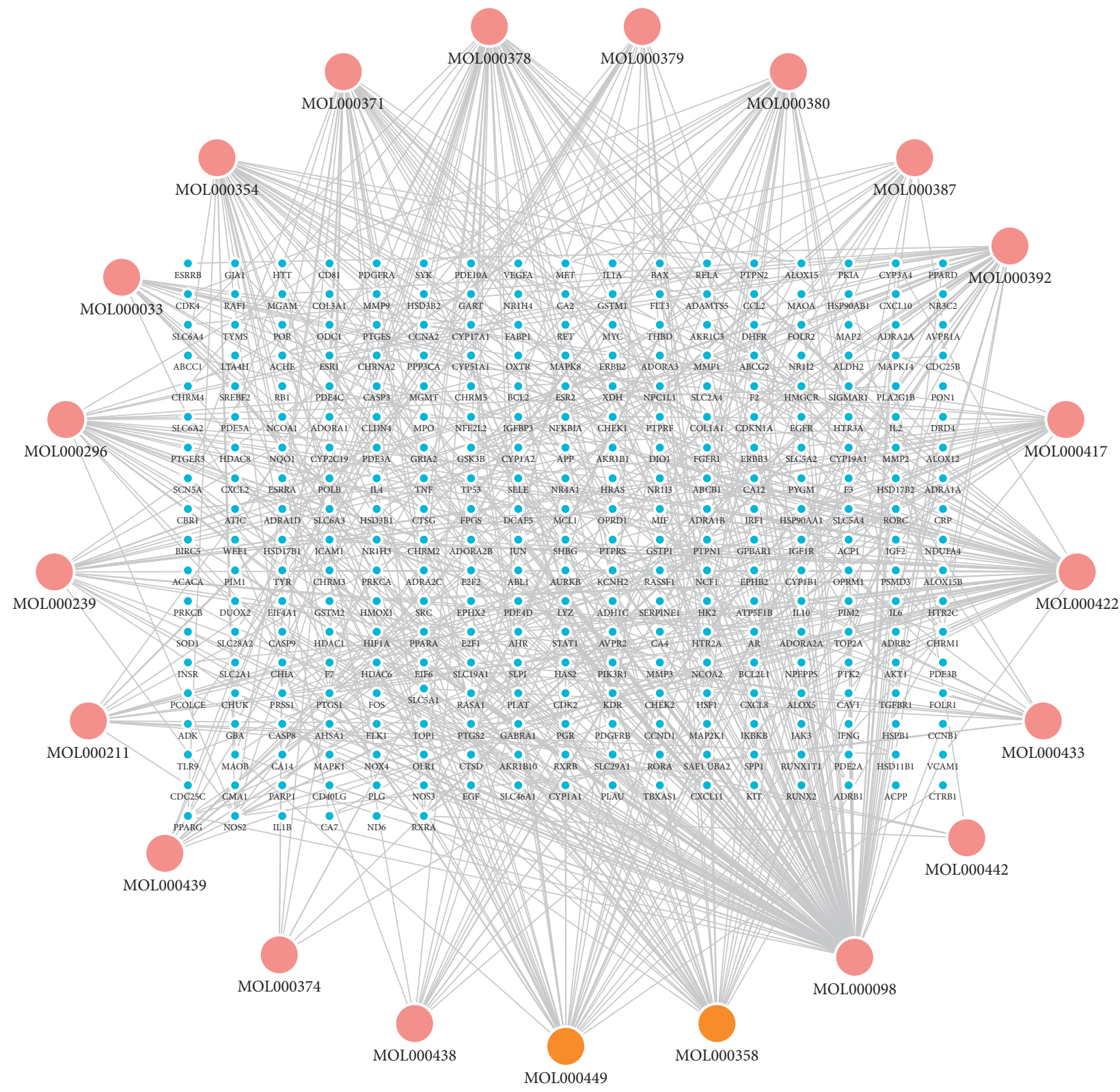

FIGURE 1: Active compounds-targets network diagram. Light blue represents targets, pink represents active compounds of Radix Astragali, and orange represents active compounds of Radix Angelicae sinensis.

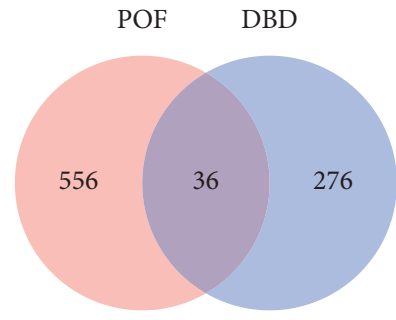

FIGURE 2: Venn diagram of DBD and the intersection genes in POF. DBD: Danggui Buxue Decoction; POF, premature ovarian failure.

ovarian steroidogenesis, etc. In the study, the top 20 signal pathways were selected according to the $P$-value to map a path bubble diagram, as shown in Figure 6.
3.6. Molecular Docking. The protein receptors and active compounds corresponding to TP53 (PID: 4CZ7), IL6 (PID: 1ALU), ESR1 (PID: 1UOM), AKT1 (PID: 1H10), and AR (PID: 1T73) were selected from the RSCB PDB database for molecular docking. The docking results of the 2 active core compounds and the 5 core protein receptors were all less than $-5.0 \mathrm{~kJ} / \mathrm{mol}$, suggesting that the active core compounds in the $\mathrm{DBD}$ and core targets in POF had good binding capacity, as shown in Table 3 and Figure 7.

3.7. Chromatogram. In the chromatographic analysis, the retention time of the $\mathrm{DBD}$ sample is consistent with the retention time of the standard product, indicating that the 


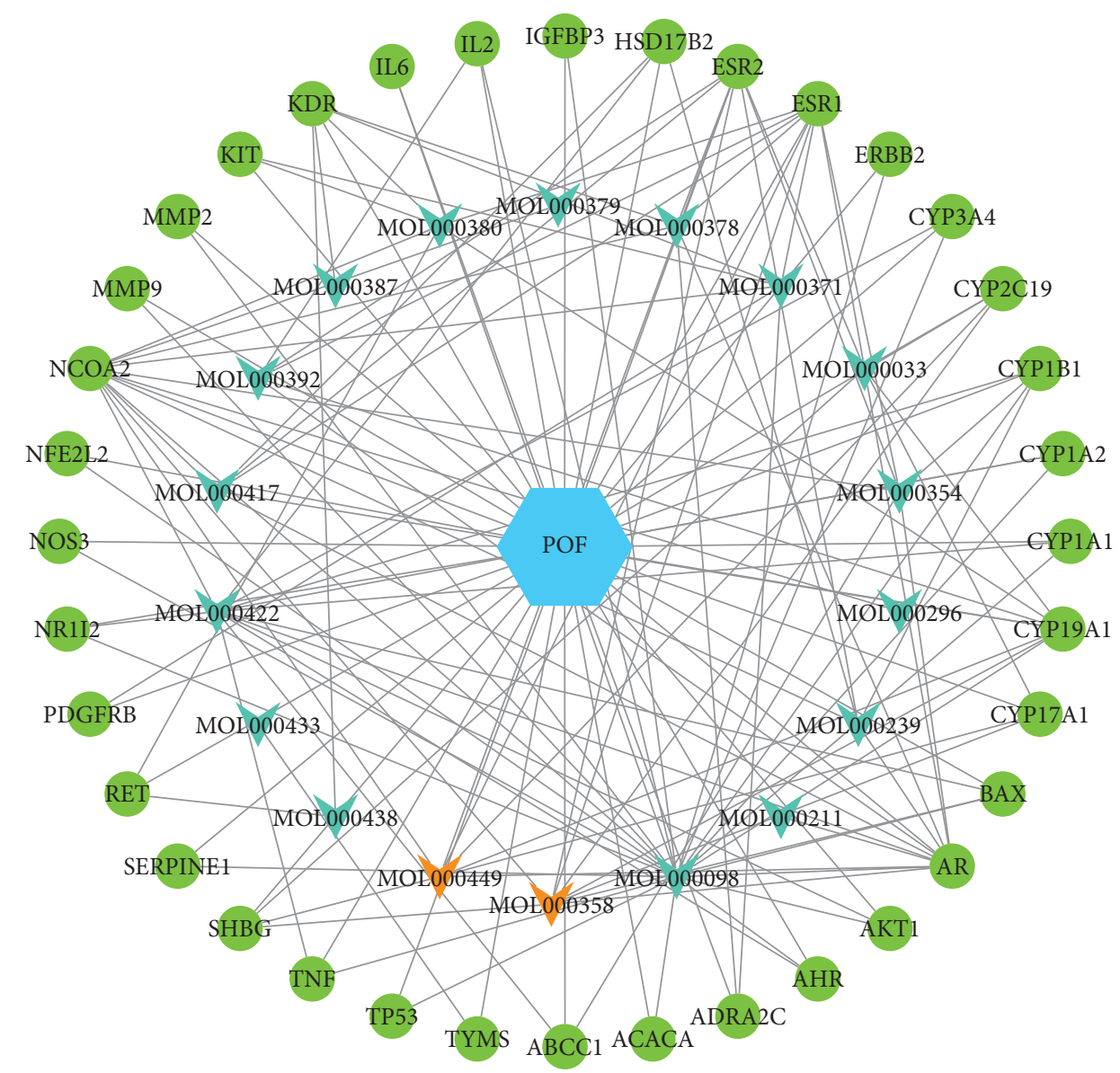

Figure 3: Active compounds-POF-targets network diagram. The light green circles represent targets, green V shapes represent Radix Astragali, orange V shapes represent Radix Angelicae sinensis, and the blue hexagon represents POF.

TABLE 1: The core active compounds with a high degree value in the active compounds-POF-targets network.

\begin{tabular}{lccc}
\hline Compound & Degree & Stress & Topological coefficient \\
\hline Quercetin & 23 & 1980 & 0.1826087 \\
Kaempferol & 13 & 736 & 0.25128205 \\
\hline
\end{tabular}

sample contains two compounds, quercetin and kaempferol, as shown in Figure 8.

3.8. Estrous Cycles of Rats in Each Group. Compared to control group, the estrus cycle of rats in the POF model group disordered. Compared to model group, estrus cycle in DBD group, quercetin group, and kaempferol group improved $(P<0.05)$, as shown in Figure 9.

3.9. Organ Index of Rats in Each Group. Compared to control group, the uterine index and the ovary index of rats in the POF model group decreased $(P<0.05)$. DBD group, quercetin group, and kaempferol group showed that ovary index increased compared to model group $(P<0.05)$ and DBD group showed that the uterine index increased compared to model group $(P<0.05)$, as shown in Figure 10 .
3.10. The Level of $E_{2}, F S H, A M H$, and $L H$ in Serum. Compared to control group, while the levels of $\mathrm{E}_{2}$ and $\mathrm{AMH}$ were significantly diminished, the levels of FSH and LH were significantly increased in the serum of model group rats $(P<0.05)$. Compared to model group, the levels of $\mathrm{E}_{2}$ were significantly increased, while the levels of LH and FSH were significantly diminished in rats serum of DBD group, quercetin group, and kaempferol group $(P<0.05)$ and the levels of AMH were significantly increased in the rats serum of DBD group and quercetin group $(P<0.05)$, as shown in Figure 11.

3.11. Protein Expression Levels of p53, IL6, Cytochrome C, $B A X, A K T 1$, ESR1, AR, and BCL2 in Ovarian Tissue. Compared to control group, the expression of p53, IL6, Cytochrome $\mathrm{C}$, and BAX increased significantly, the expression of AKT1, ESR1, AR and BCL2 decreased significantly in the ovaries tissue of rats in the POF model group $(P<0.05)$. Compared to model group, the expression of AKT1 and ESR1 was significantly increased in DBD group, quercetin group, and kaempferol group, the expression of Cytochrome C was significantly decreased in DBD group, quercetin group, and kaempferol group, the expression of p53 and IL6 was significantly decreased in quercetin group 


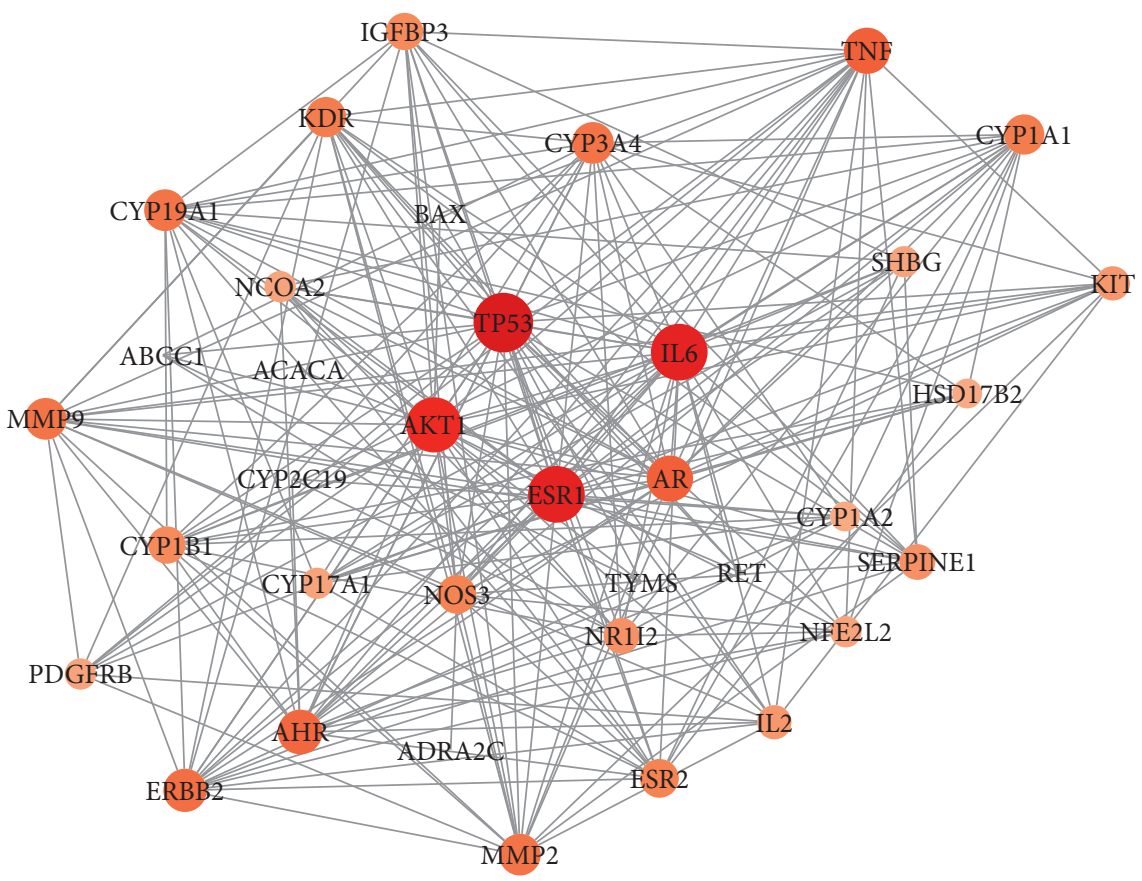

(a)

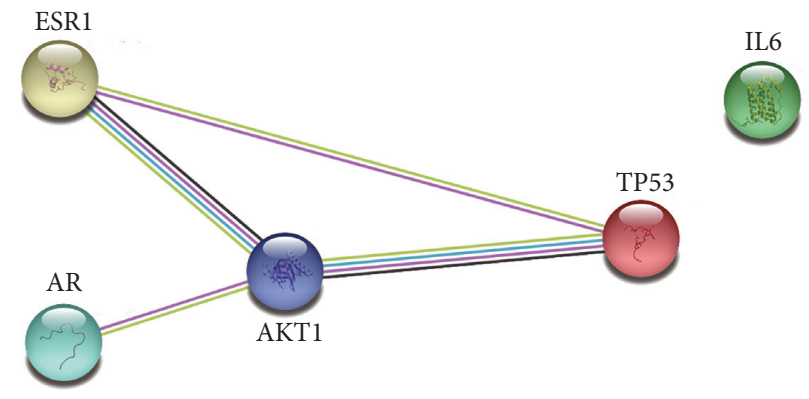

(b)

FIgURE 4: The PPI network of DBD-intermediating POF targets. (a) The PPI network of 36 targets of DBD intervening POF targets. The size and depth of the node color represent the scale of the degree value. (b) The PPI network of five core targets.

TABLE 2: Topological parameters of the core targets in PPI.

\begin{tabular}{lccc}
\hline Gene symbol & Degree & Stress & Topological coefficient \\
\hline TP53 & 28 & 676 & 0.39591837 \\
ESR1 & 26 & 534 & 0.41758242 \\
IL6 & 26 & 462 & 0.42527473 \\
AKT1 & 25 & 604 & 0.40235294 \\
AR & 19 & 254 & 0.48297214 \\
\hline
\end{tabular}

and DBD group, the expression of BCL2 was significantly increased in quercetin group and $\mathrm{DBD}$ group, the expression of AR was increased significantly in DBD group, and the expression of BAX was decreased significantly in DBD group $(P<0.05)$, as shown in Figure 12.

\section{Discussion}

POF is a female reproductive system disease resulting from loss of ovarian function induced by autoimmune diseases, neurological disorders, iatrogenic injuries, bad lifestyle habits, and other factors. CTX exerts a certain toxicity on the gonad, accelerating the maturation of the primordial ovarian follicles into mature follicles. Meanwhile, long-term use or high-dose CTX can reduce the ovarian reserve in female patients [31]. Studies have shown that DBD could repair ovarian injury in rats by reducing the expression of ovarian CYP24A1 mRNA and increasing the expression of serum $\mathrm{E}_{2}$ and ovarian ERa mRNA [30]. DBD could have broad application prospects for the treatment of POF, and so it is of certain practical significance to carry out basic experimental research on it [32].

The analysis of the PPI network suggested that the process of intervening POF for DBD is highly correlated with TP53, IL6, ESR1, AKT1, AR, and other genes. As a gene 


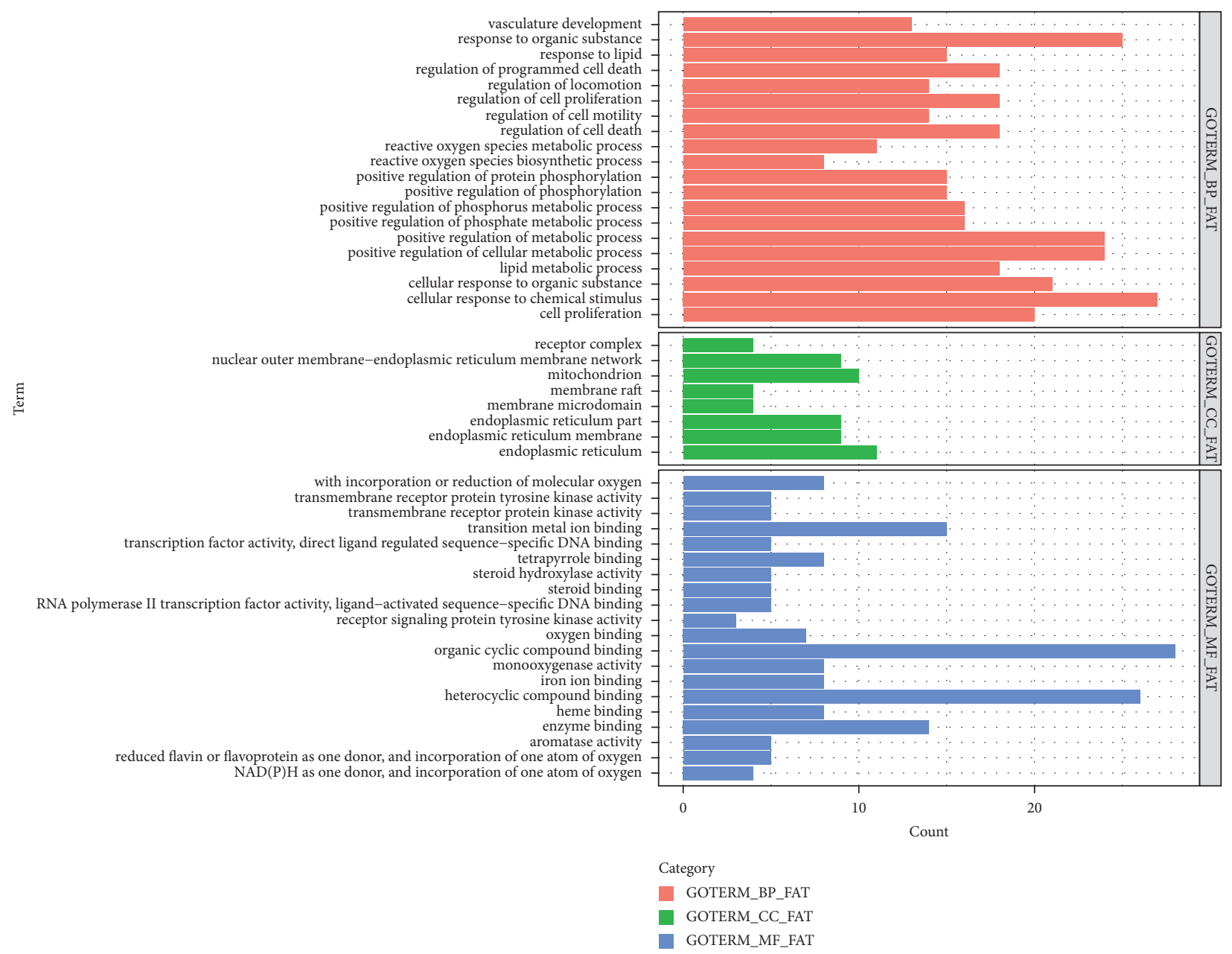

FIGURE 5: The histogram of the GO function of the POF targets mediated by DBD. The red indicates biological processes (BP), green indicates cell compositions (CC), and blue indicates molecular functions (MF).

related to senility, TP53 can induce the arrest of the development or apoptosis of the oocyte, thus accelerating the process of POF [33]. IL6 can not only induce B cell proliferation and $\mathrm{T}$ cell proliferation to produce an inflammatory response, but also increase the risk of $\mathrm{POF}$ complications and reduce the expression level of $\mathrm{E}_{2}$, testosterone (T), and ovarian volume (OV) [34]. The polymorphism at the ESR1 site affects the development process of POF, among which the polymorphism at the PvuII (rs2234693) site can induce many complications, such as osteoporosis and cardiovascular disease [35]. However, AKT1 phosphorylation is able to improve the fertility of POF model mice because it can promote the growth rate of secondary follicles and small follicles in the ovary and enhance the function of ovarian to secrete sex hormones [36]. By inducing the differentiation of primordial follicles into primary follicles, secondary follicles, and mature follicles by regulating $\mathrm{AR}$, androgen is capable of regulating paracrine factors and reducing follicular atresia [37].

The GO and KEGG enrichment analysis showed that the reactive oxygen species metabolic process and release of Cytochrome $\mathrm{C}$ from mitochondria and apoptotic signaling pathway, p53 signaling pathway, the PI3K-Akt signaling pathway, and the estrogen signaling pathway play important roles in the intervention of DBD to POF. Persistent upregulation of ROS is the major feature of oxidative stress in cells and is an important factor causing cell aging [38]. Numerous studies have shown that GC apoptosis accelerates follicular atresia leading to POF [39]. A study has been shown to promote the release of Cytochrome $\mathrm{C}$ into the cytosol, which could lead to upregulation of cytochrome $\mathrm{C}$ in POF rat ovarian tissue [40]. The p53 signaling pathway can reduce the ovarian and uterine index and increase serum levels of FSH, LH, and the number of oocytes in the ovary by inhibiting the expression of $\mathrm{Mdm} 2$ and Mdm4 [41]. By regulating the PI3K/AKT signaling pathways, the sex hormone levels are effectively regulated, pathological injuries are relieved in POF rats, such as ovarian atrophy, cortical thickening, and structural disturbances, and the activation and maturation of ovarian granulosa cells are also promoted [42]. The estrogen signaling pathway is closely related to POF. Low estrogen level may cause estrous cycle disturbance and decreased ovarian function and spatial cognitive ability, as well as abnormal mental states in mice such as anxiety and depression [43]. The molecular docking results further verified the active compounds and potential targets 


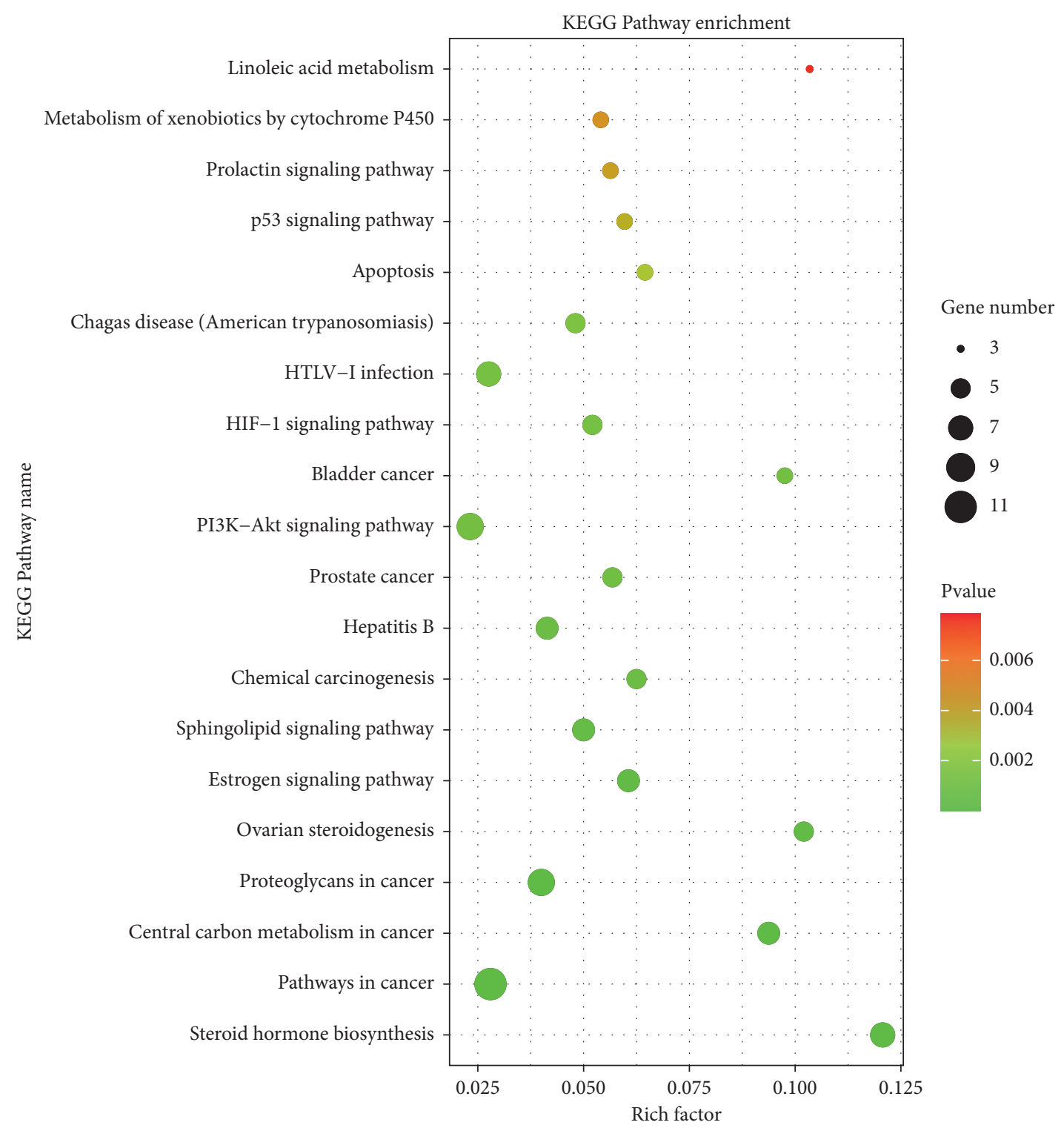

Figure 6: KEGG pathway bubble chart of DBD intervening POF targets.

TABle 3: The binding energy of core active compounds and core protein receptors.

\begin{tabular}{lccccc}
\hline \multirow{2}{*}{ Compound } & \multicolumn{5}{c}{ Binding energy/(kJ/mol) } \\
& TP53 & IL6 & ESR1 & AKT1 & AR \\
\hline Quercetin & -32.23 & -27.21 & -35.58 & -24.70 & -36.42 \\
Kaempferol & -29.72 & -25.53 & -34.74 & -24.28 & -36.00 \\
\hline
\end{tabular}

predicted by network pharmacology and found that quercetin and kaempferol in DBD and the five core targets (TP53, IL6, ESR1, AKT1, and AR) have good results as regards the binding energy.

Based on the network database and bioinformatics results in this study, we found that the mechanism of DBD and its effective compounds in improving POF may be related to the balance of ESR and AR in the TP53-AKT signaling pathway. In the TP53-AKT signaling pathway, TP53 and AKT1 have the characteristics of mutual antagonists and play two opposite biological functions [44, 45]. TP53 can affect cell cycle arrest, apoptosis, and senescence by regulating the AKT pathway [46]. The balance of ESR and AR plays an important role in regulating the reproductive system, promoting ovarian development, and maintaining reproductive function [47]. Akt phosphorylation and inactivation can induce AR-dependent proliferative response and then participate in the process of regulating cell growth proteins [48]. The AKT can inhibit ESR-positive breast cancer cell proliferation [49]. Translocation of p-Akt may be related to suppression of estrogen-induced apoptosis in endometrial carcinogenesis [50]. DBD had a regulatory effect on the TP53-AKT signaling pathway and could inhibit the BAX/BCL2 ratio by activating Akt phosphorylation and estrogen receptors [51]. And by regulating BCL2 family proteins, DBD could inhibit cell apoptosis, promote the development of antral follicles, increase AMH secretion, and save ovarian function [32]. Quercetin and kaempferol, as the 

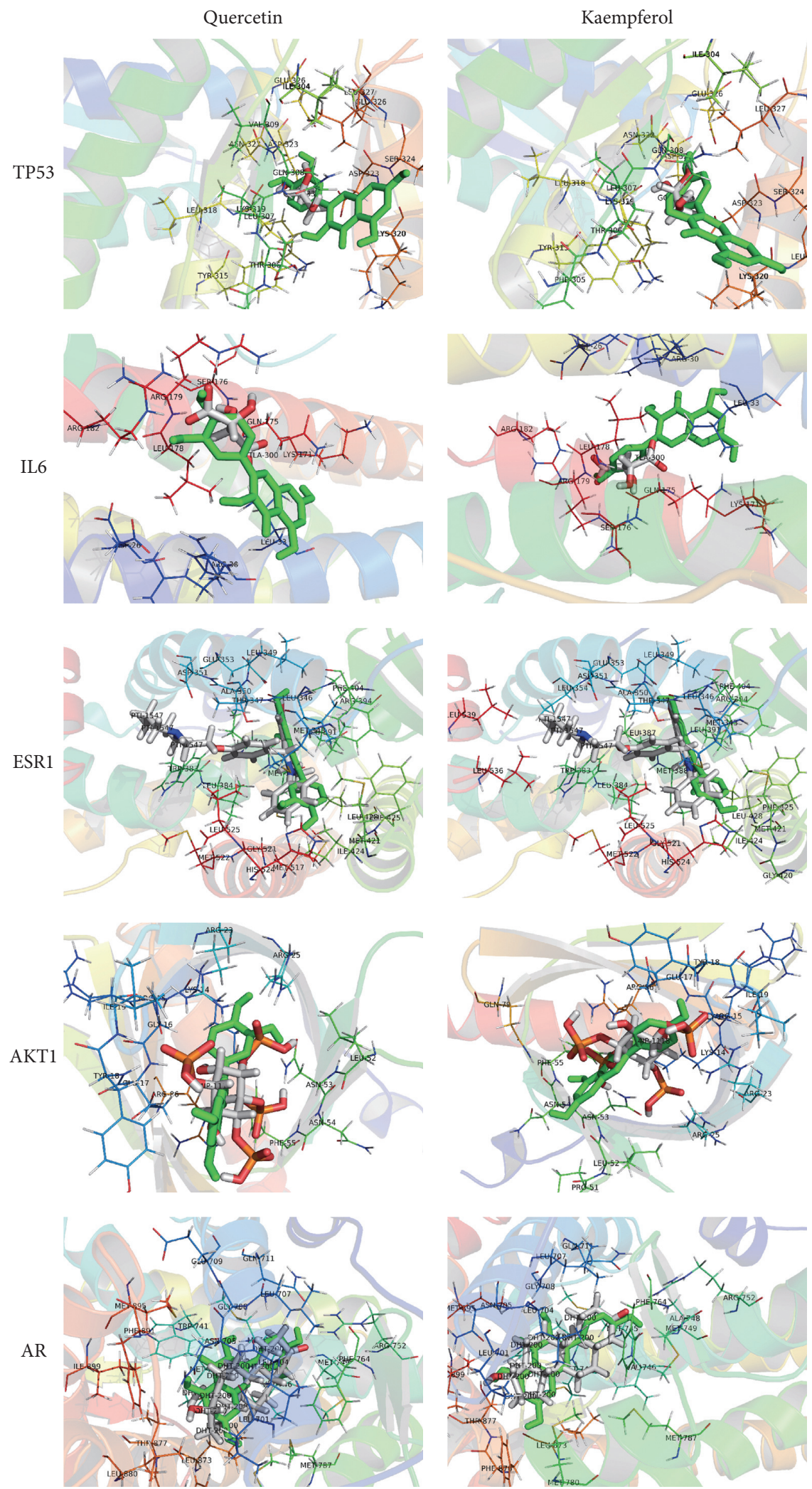

FIGURE 7: Molecular docking diagram of the core active compounds and the core targets.

main active compounds of $\mathrm{DBD}$, also have a certain therapeutic effect on common gynecological diseases. Quercetin is a pentahydroxy flavonol that could regulate the response to physical oxidative stress and the response to inflammation, increase the number of ovarian follicles and the level of estrogen expression in patients, improve the function of the 

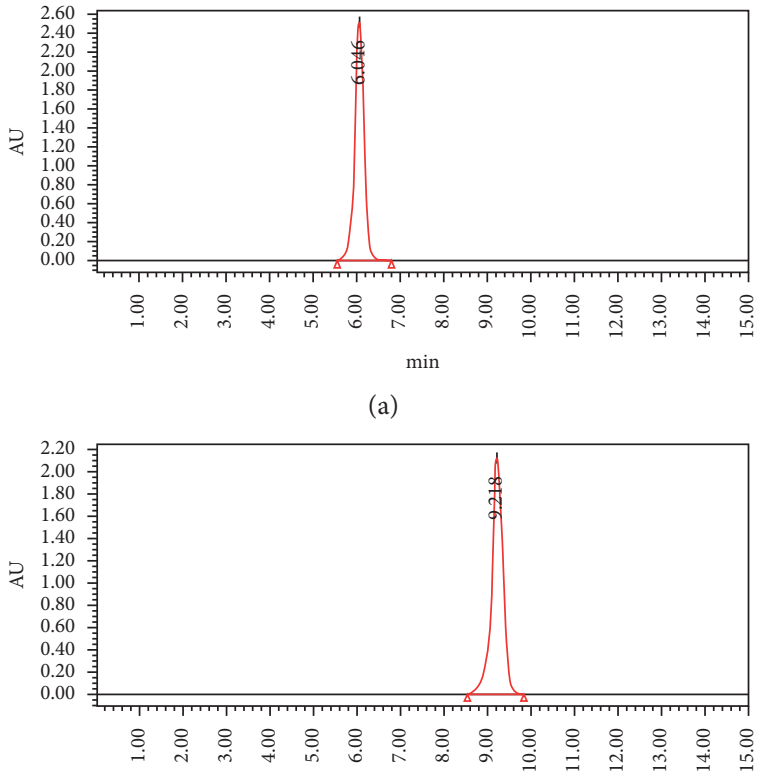

(c)

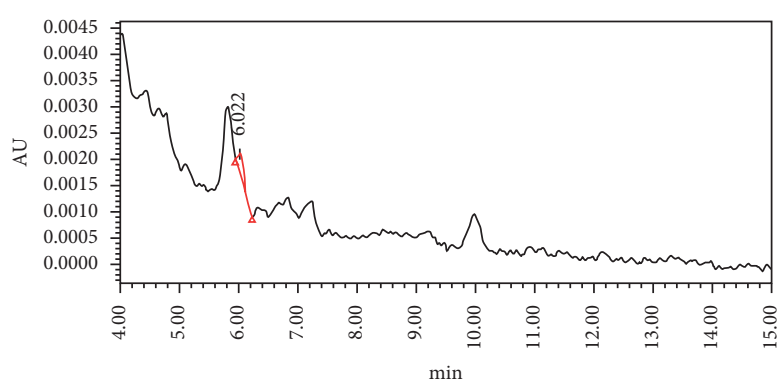

(b)

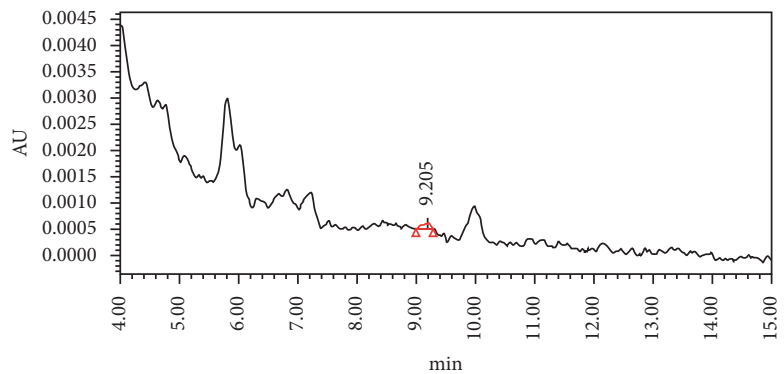

(d)

FIgURE 8: (a) Standard quercetin chromatogram. (b) Quercetin chromatogram in DBD. (c) Standard kaempferol chromatogram. (d) Kaempferol chromatogram in DBD.

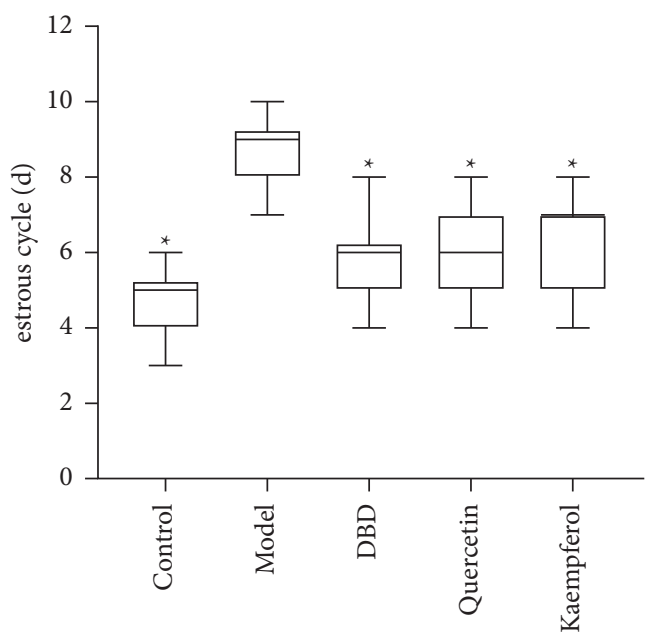

FIGURE 9: Effect of DBD on the estrous cycle in the rat POF model $(n=10) .{ }^{*} P<0.05$, compared with model group.

ovarian reserve function, and gradually restore the patient's fertility [52]. Quercetin can also inhibit the proliferation of MCF-7 cells and attenuate mammary cancer by regulating TP53-AKT signaling pathway [53]. Kaempferol can regulate the levels of p53, AKT1, BAX, and BCL-X protein in the TP53-AKT signaling pathway of ovarian cancer cells, leading to apoptosis of ovarian cancer cells $[54,55]$.

Through the above situation, we used animal experiments to initially explore the regulation of $\mathrm{DBD}$ to regulate the balance of ESR and AR in the TP53-AKT signaling pathway, thereby preventing the molecular mechanism of treatment of POF. Research results show that DBD and its effective compounds can restore the estrus cycle, increase ovarian index, uterine index, serum $\mathrm{E}_{2}, \mathrm{AMH}$ levels, and ovarian
AKT1, ESR1, AR, and BCL2 protein expression levels, reduce serum FSH and LH levels, ovarian p53, IL6, Cytochrome C, and BAX protein expression levels of POF model rats, and have a good therapeutic effect on POF. In comparison, DBD has the most comprehensive regulatory targets and the best effect, while quercetin and kaempferol have similar but different key targets in the treatment of POF. For example, the effect of quercetin and kaempferol on ESR1 is close to DBD. The effect of DBD on AR is better than quercetin and kaempferol. This suggests that traditional Chinese medicine and its effective compounds have their own advantages, and dialectical use may provide a better treatment for POF. This had also been verified in the research process of the treatment of asthma by Schisandra chinensis [56]. 


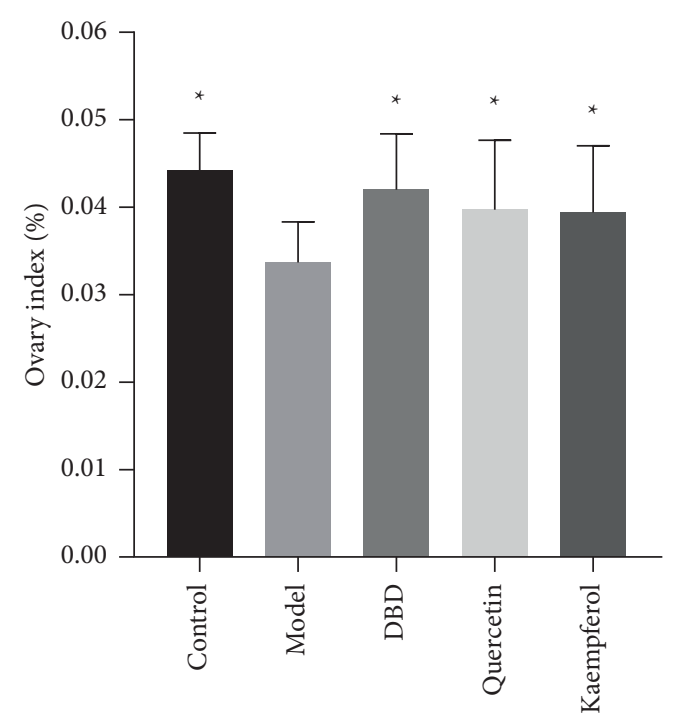

(a)

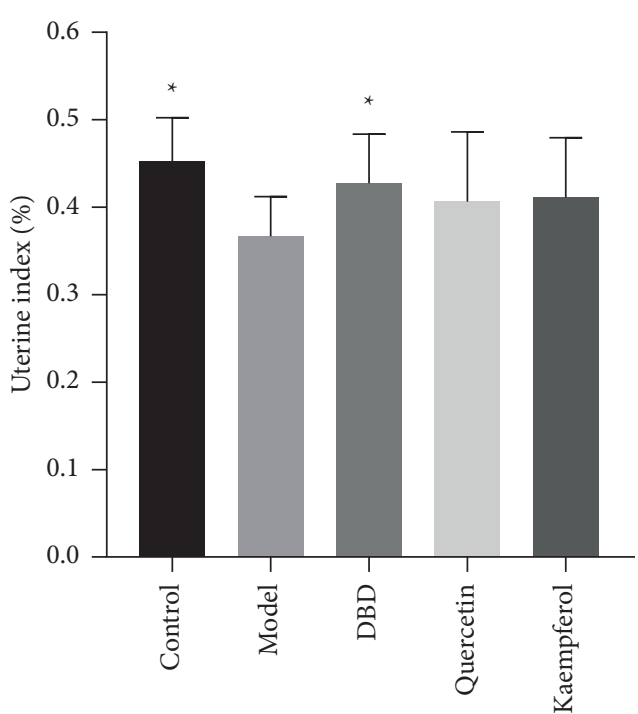

(b)

FIGURe 10: Effect of DBD on organ index in rat POF model. (a) Ovary index $(n=10)$. (b) Uterine index $(n=10) .{ }^{*} P<0.05$, compared to the model group.

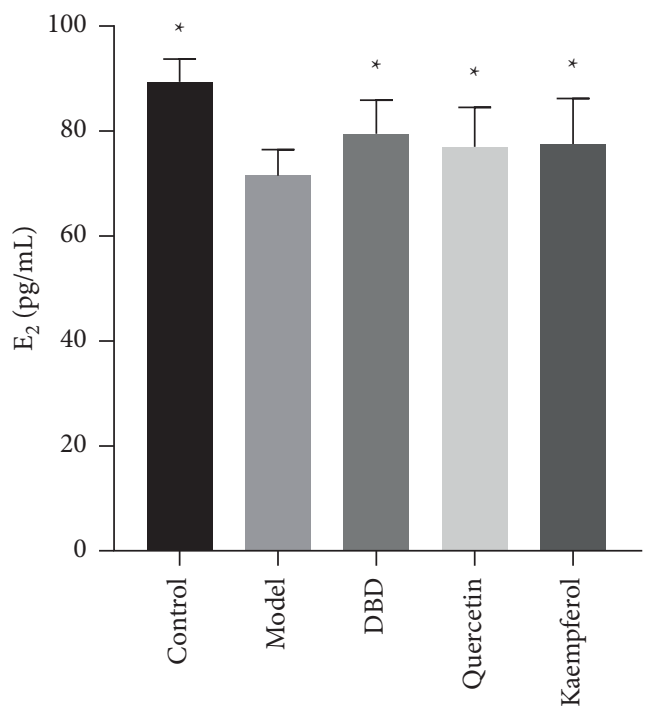

(a)

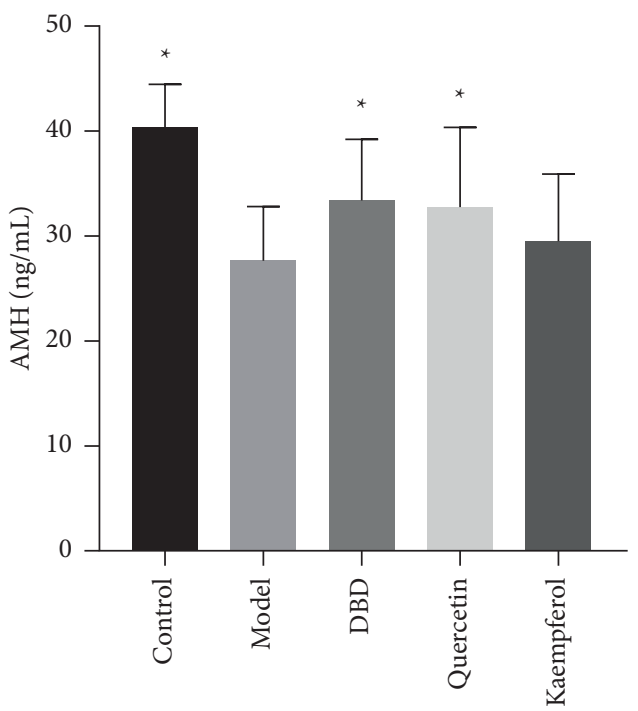

(b)

FIGURE 11: Continued. 


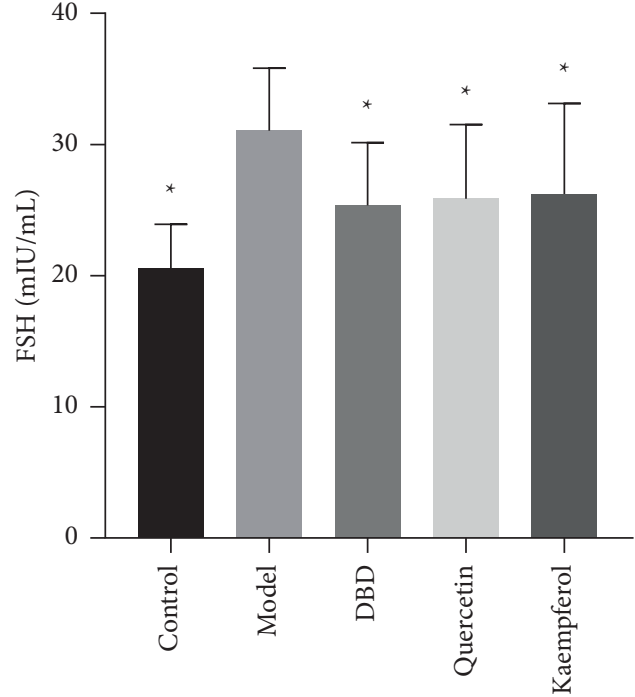

(c)

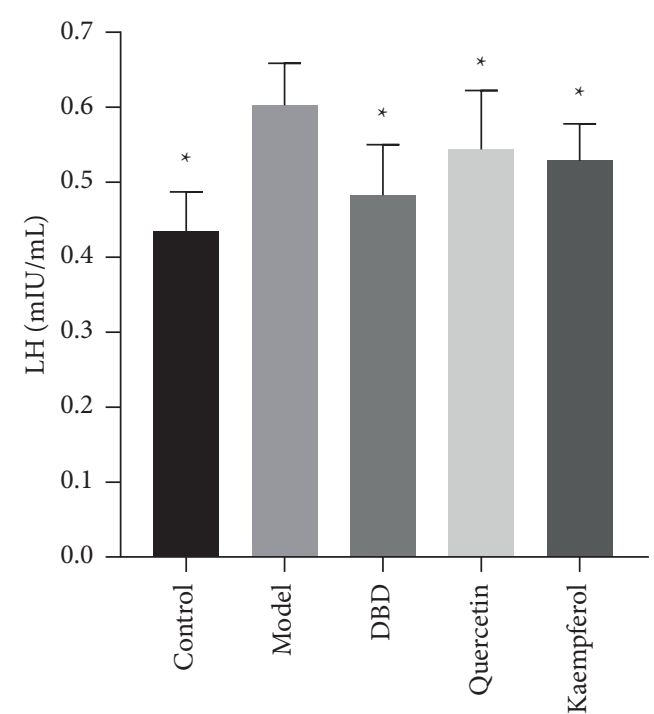

(d)

Figure 11: Effect of DBD on serum levels of $\mathrm{E}_{2}, \mathrm{FSH}, \mathrm{AMH}$, and $\mathrm{LH}$ in POF model rats. (a) Serum estradiol $\left(\mathrm{E}_{2}\right)(n=10)$. (b) Serum antimullerian hormone $(\mathrm{AMH})(n=10)$. (c) Serum follicular stimulating hormone (FSH) $(n=10)$. (d) Serum luteinizing hormone (LH) $(n=10) .{ }^{*} P<0.05$, compared to model group.

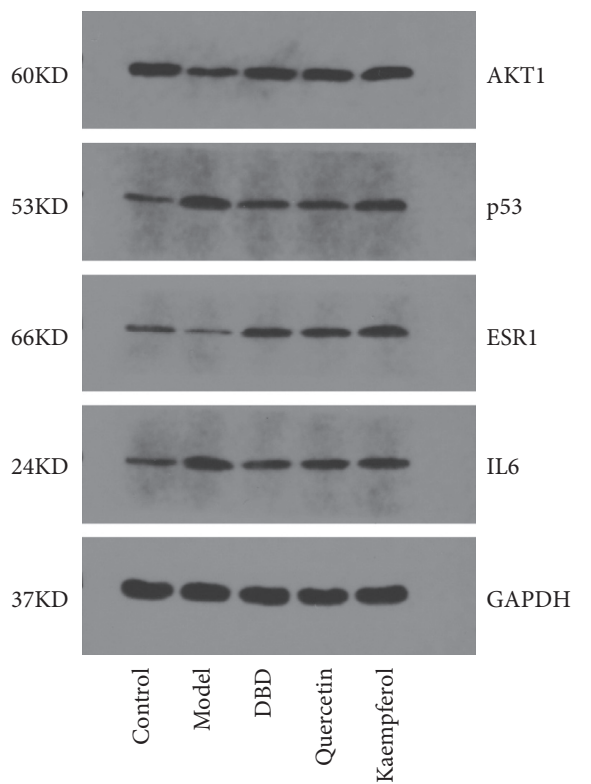

(a)

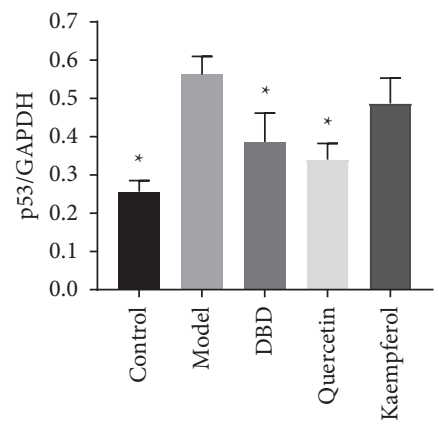

(d)
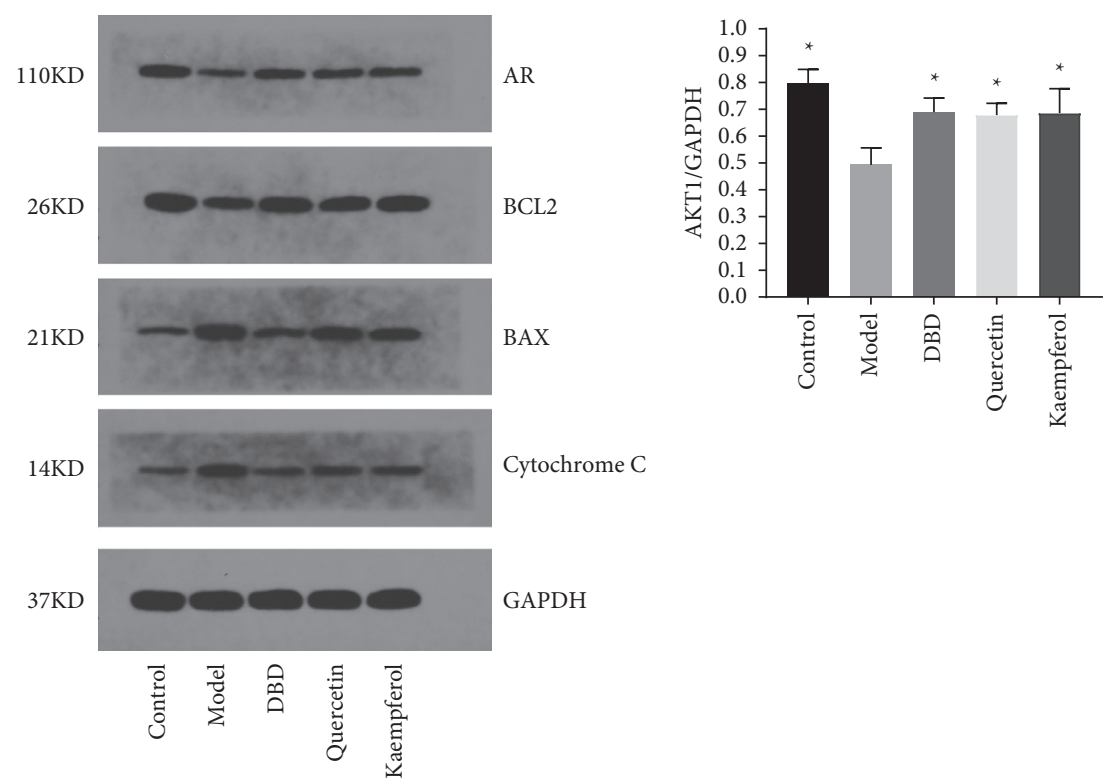

(b)

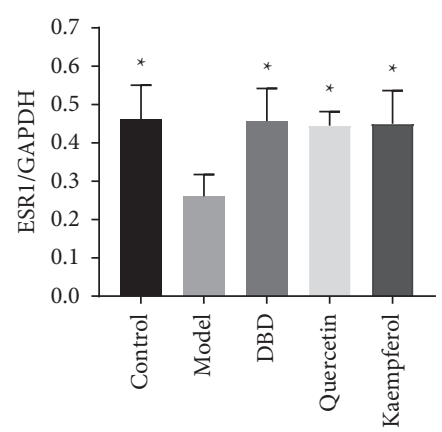

(e) (c)

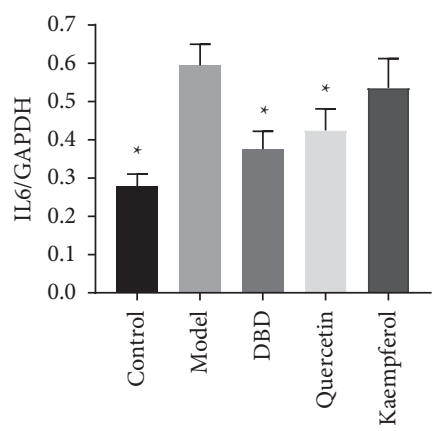

(f)

Figure 12: Continued. 


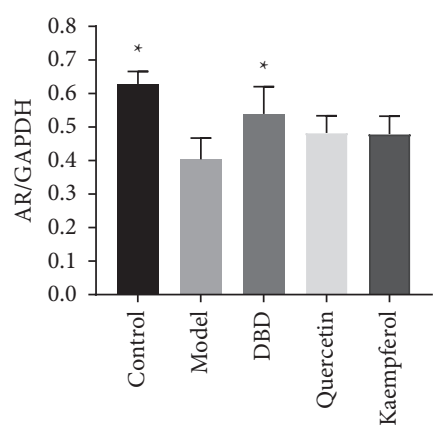

(g)

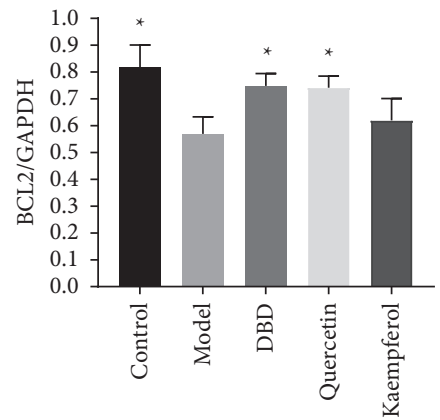

(h)

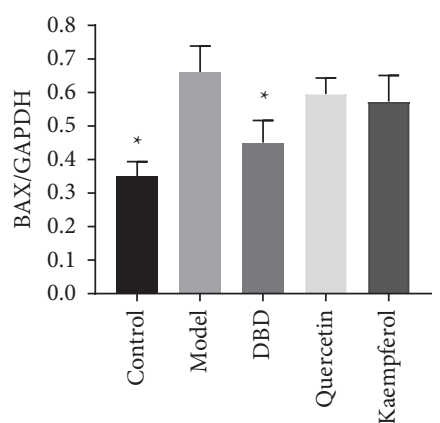

(i)

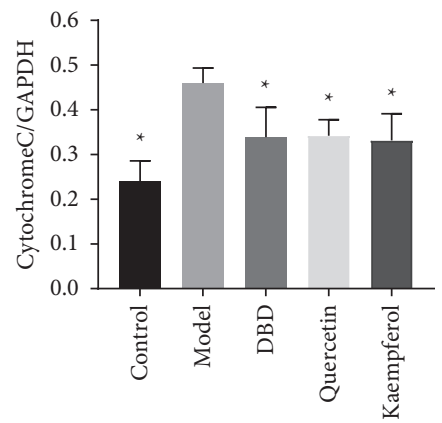

(j)

FIGURE 12: Effects of DBD on the expression of AKT1, p53, ESR1, IL6, AR, BCL2, BAX, and Cytochrome C proteins in ovary tissues. (a) Western blot analysis of the proteins AKT1, p53, ESR1, and IL6 proteins in the ovaries tissue. (b) Western blot analysis of the proteins AR, BCL2, BAX, and Cytochrome C in the ovary tissue; (c-j): Protein expressions of AKT1, p53, ESR1, IL6, AR, BCL2, BAX, and Cytochrome $\mathrm{C}(n=3) ;{ }^{*} \mathrm{P}<0.05$, compared to model group.

\section{Conclusions}

We found that DBD and its active components could treat POF by regulating the balance of ESR and AR in the TP53AKT signaling pathway. However, due to the delay in updating the information recorded in the TCM, disease, and bioinformatics databases, certain critical targets or pathways can be overlooked. In the future, our teams will establish more studies in combination with transcriptomics, metabolomics, and proteomics to more fully explain the molecular mechanism of DBD during POF intervention.

\section{Data Availability}

The data that support the findings of this study are available from the corresponding author upon reasonable request.

\section{Disclosure}

Huaiquan Li and Hong Yang are co-first authors.

\section{Conflicts of Interest}

The authors report that there are no conflicts of interest.

\section{Authors' Contributions}

Conception and design of the research were carried out by MZ, JC, HL, and HY; acquisition of data was performed by
HY, WL, YC, XZ, and JC; analysis and interpretation of data were done by HY, WL, YC, ZQ, and JC; statistical analysis was performed by $\mathrm{HY}, \mathrm{WL}, \mathrm{YC}, \mathrm{XZ}$, and JC; funding was acquired by MZ, JC, and HL; manuscript was drafted by HL and HY; revision of manuscript for important intellectual content was done by MZ and JC. All the authors read and approved the final manuscript.

\section{Acknowledgments}

This work was supported by the Three-Year Action Plan for the Development of Shanghai Traditional Chinese Medicine (nos. ZY[2018-2020]-FWTX-6006 and ZY[2018-2020]-FWTX4005) and the Science and Technology Research Project of Jiangxi Provincial Department of Education (no. GJJ200256).

\section{References}

[1] S. Vujović, M. Ivović, M. Tancić-Gajić et al., "Premature ovarian failure," Srpski Arhiv za Celokupno Lekarstvo, vol. 140, no. 11-12, pp. 806-811, 2012.

[2] Practice Committee of the American Society for Reproductive Medicine, "Testing and interpreting measures of ovarian reserve: a committee opinion," Fertility and Sterility, vol. 114, no. 6, pp. 1151-1157, 2020.

[3] A. Podfigurna-Stopa, A. Czyzyk, M. Grymowicz et al., "Premature ovarian insufficiency: the context of long-term effects," Journal of Endocrinological Investigation, vol. 39, no. 9, pp. 983-990, 2016. 
[4] J. Lin, X.-L. Li, H. Song et al., "A general description for Chinese medicine in treating premature ovarian failure," Chinese Journal of Integrative Medicine, vol. 23, no. 2, pp. 91-97, 2017.

[5] H. F. Li, Q. H. Shen, W. J. Chen, W. M. Chen, Z. F. Feng, and L. Y. Yu, "Efficacy of traditional Chinese medicine tonifying kidney (bushen) and activating blood (huoxue) prescription for premature ovarian insufficiency: a systematic review and meta-analysis," Evidence-based Complementary and Alternative Medicine, vol. 2020, Article ID 1789304, 2020.

[6] S. Hossain, Z. Urbi, H. Karuniawati et al., "Andrographis paniculata (burm. F.) wall. Ex nees: an updated review of phytochemistry, antimicrobial pharmacology, and clinical safety and efficacy," Life (Basel, Switzerland), vol. 114 pages, 2021.

[7] A. Rossi, E. Caiazzo, R. Bilancia et al., "Salvinorin A inhibits airway hyperreactivity induced by ovalbumin sensitization," Frontiers in Pharmacology, vol. 7, p. 525, 2016.

[8] M. N. Uddin Chy, M. Adnan, M. R. Chowdhury et al., "Central and peripheral pain intervention by Ophiorrhizarugosa leaves: potential underlying mechanisms and insight into the role of pain modulators," Journal of Ethnopharmacology, vol. 276, Article ID 114182, 2021.

[9] D. Ağagündüz, M. N. Çelik, M. E. Çıtar Dazıroğlu, and R. Capasso, "Emergent drug and nutrition interactions in COVID-19: a comprehensive narrative review," Nutrients, vol. 135 pages, 2021.

[10] M. M. Rahman, A. S. M. A. Reza, M. A. Khan et al., "Unfolding the apoptotic mechanism of antioxidant enriched-leaves of Tabebuia pallida (lindl.) miers in EAC cells and mouse model," Journal of Ethnopharmacology, vol. 278, Article ID 114297, 2021.

[11] J. N. R. Moni, M. Adnan, A. M. Tareq et al., A. H. M. Khurshid Alam, S. Binte Alam, M. Ahmed Sakib, Ki Kwang Oh, D. Ha Cho, and R. Capasso, Therapeutic potentials of fruit (seed) reflected into an array of pharmacological assays and prospective receptors-mediated pathways," Life (Basel, Switzerland), vol. 112 pages, 2021.

[12] E. K. Akkol, I. I. Tatlı, G. . Ş. Karatoprak et al., "Is emodin with anticancer effects completely innocent? Two sides of the coin," Cancers, vol. 1311 pages, 2021.

[13] N. S. Macêdo, Z. d. S. Silveira, A. H. Bezerra et al., "(Fabaceae) phytochemistry, ethnobotany, and bioactivities: a review," Molecules, vol. 2517 pages, 2020.

[14] I. L. I. Hook, "Danggui to Angelica sinensis root: are potential benefits to European women lost in translation? A review," Journal of Ethnopharmacology, vol. 152, no. 1, pp. 1-13, 2014.

[15] H. Q. Lin, A. G. W. Gong, H. Y. Wang et al., "Danggui buxue tang (astragali Radix and Angelicae sinensis Radix) for menopausal symptoms: a review," Journal of Ethnopharmacology, vol. 199, pp. 205-210, 2017.

[16] X.-Q. Shi, S.-J. Yue, Y.-P. Tang et al., "A network pharmacology approach to investigate the blood enriching mechanism of Danggui buxue Decoction," Journal of Ethnopharmacology, vol. 235, pp. 227-242, 2019.

[17] Q.-F. Xie, J.-H. Xie, T. T. X. Dong et al., "Effect of a derived herbal recipe from an ancient Chinese formula, Danggui Buxue Tang, on ovariectomized rats," Journal of Ethnopharmacology, vol. 144, no. 3, pp. 567-575, 2012.

[18] Y. Zheng, Y. Jin, H. B. Zhu, S. T. Xu, Y. X. Xia, and Y. Huang, "Effects of a Chinese medicinal plant Radix astragali on the ovariectomized female rats," African Journal of Traditional, Complementary and Alternative Medicines, vol. 10, no. 1, pp. 9-14, 2012.
[19] F. Zhao, Q. S. Xu, Q. A. Yuan, and N. Li, "Medicated serum of Danggui buxue tang has effect on the expression of caspase-3 protein of ovarian granulosa cells in rats with cisplatin injury," Journal New Chinese Medical, vol. 50, no. 07, pp. 8-11, 2018.

[20] Y. Jiang, M. Zhong, F. Long, and R. Yang, "Deciphering the active ingredients and molecular mechanisms of tripterygium hypoglaucum (levl.) hutch against rheumatoid arthritis based on network pharmacology," Evidence-based Complementary and Alternative Medicine, vol. 2020, Article ID 2361865, 2020.

[21] D. Gan, X. Xu, D. Chen, P. Feng, and Z. Xu, "Network pharmacology-based pharmacological mechanism of the Chinese medicine rhizoma drynariae against osteoporosis," Medical Science Monitor, vol. 25, pp. 5700-5716, 2019.

[22] J. Huang, H. Tang, S. Cao et al., "Molecular targets and associated potential pathways of danlu capsules in hyperplasia of mammary glands based on systems pharmacology," Evidence-based Complementary and Alternative Medicine, vol. 2017, Article ID 1930598, 2017.

[23] L. Ge, K. Cheng, and J. Han, "A network pharmacology approach for uncovering the osteogenic mechanisms of Psoralea corylifolia linn," Evidence-based Complementary and Alternative Medicine, vol. 2019, Article ID 2160175, 2019.

[24] J. Zhou, Q. Wang, Z. Xiang et al., "Network pharmacology analysis of traditional Chinese medicine formula xiao ke yin shui treating type 2 diabetes mellitus," Evidence-based Complementary and Alternative Medicine, vol. 2019, Article ID 4202563, 2019.

[25] G. Xie, W. Peng, P. Li et al., "A network pharmacology analysis to explore the effect of astragali radix-radix Angelica sinensis on traumatic brain injury," BioMed Research International, vol. 2018, Article ID 3951783, 2018.

[26] Y. Zong, M. Ding, S. T. Ma, and W. Ju, "Investigation of potential Chinese materia medica and its monomers in treatment of coronavirus disease 2019 (COVID-19) based on angiotensin converting enzyme II (ACE2) receptor," Chinese Traditional and Herbal Drugs, vol. 51, no. 05, pp. 1123-1129, 2020.

[27] R. Melekoglu, O. Ciftci, S. Eraslan, A. Cetin, and N. Basak, "Beneficial effects of curcumin and capsaicin on cyclophosphamide-induced premature ovarian failure in a rat model," Journal of Ovarian Research, vol. 11, no. 1, p. 33, 2018.

[28] M. Jafari Khorchani, F. Zal, and A. Neisy, "The phytoestrogen, quercetin, in serum, uterus and ovary as a potential treatment for dehydroepiandrosterone-induced polycystic ovary syndrome in the rat," Reproduction, Fertility and Development, vol. 32, no. 3, pp. 313-321, 2020.

[29] X. Wang, S. Yang, L. Cao, and Z. Zhang, "Protective effects of kaempferol on bone collagen production and trabecular bone loss in ovariectomized rats," Chinese Journal of Clinical Pharmacology, vol. 36, no. 10, pp. 1302-1305, 2020.

[30] M. Zhang, L. Guan, Z. Qin et al., "Effect of Danggui buxue decoction on the expression of CYP24A1 and ER $\alpha$ in ovaries of osteoporosis rats induced by retinoic acid," Traditional Chinese Drug Research \& Clinical Pharmacology, vol. 31, no. 05, pp. 502-507, 2020.

[31] B.-f. Zhang, Y. Hu, X. Liu et al., "The role of AKT and FOXO3 in preventing ovarian toxicity induced by cyclophosphamide," PLoS One, vol. 13, no. 8, Article ID e0201136, 2018.

[32] L. Wang, J. Liu, G. Nie, Y. Li, and H. Yang, "Danggui buxue tang rescues folliculogenesis and ovarian cell apoptosis in rats with premature ovarian insufficiency," Evidence-based Complementary and Alternative Medicine, vol. 2021, Article ID 6614302, 2021. 
[33] J. A. Stanley, J. A. Arosh, R. C. Burghardt, and S. K. Banu, "A fetal whole ovarian culture model for the evaluation of CrVIinduced developmental toxicity during germ cell nest breakdown," Toxicology and Applied Pharmacology, vol. 289, no. 1, pp. 58-69, 2015.

[34] S. Sun, H. Chen, X. Zheng, C. Ma, and R. Yue, "Analysis on the level of IL-6, IL-21, AMH in patients with auto-immunity premature ovarian failure and study of correlation," Experimental and Therapeutic Medicine, vol. 16, no. 4, pp. 33953398, 2018.

[35] M. He, J. Shu, X. Huang, and H. Tang, "Association between estrogen receptora gene (ESR1) PvuII (T/C) and XbaI (A/G) polymorphisms and premature ovarian failure risk: evidence from a meta-analysis," Journal of Assisted Reproduction and Genetics, vol. 32, no. 2, pp. 297-304, 2015.

[36] K. Kawamura, Y. Cheng, N. Suzuki et al., "Hippo signaling disruption and Akt stimulation of ovarian follicles for infertility treatment," Proceedings of the National Academy of Sciences, vol. 110, no. 43, pp. 17474-17479, 2013.

[37] Q. Hu, L. Hong, M. Nie et al., "The effect of dehydroepiandrosterone supplementation on ovarian response is associated with androgen receptor in diminished ovarian reserve women," Journal of Ovarian Research, vol. 10, no. 1, p. 32, 2017.

[38] H. L. Jiang, L. Q. Cao, and H. Y. Chen, "Protective effects ROS up-regulation on premature ovarian failure by suppressing ROS-TERT signal pathway," European Review for Medical and Pharmacological Sciences, vol. 22, no. 19, pp. 6198-6204, 2018.

[39] H. Li, W. Zhao, L. Wang et al., "Human placenta-derived mesenchymal stem cells inhibit apoptosis of granulosa cells induced by IRE $1 \alpha$ pathway in autoimmune POF micee," Cell Biology International, vol. 43, no. 8, pp. 899-909, 2019.

[40] Y. Yao, Y. Xu, and Y. Wang, "Protective roles and mechanisms of rosmarinic acid in cyclophosphamide-induced premature ovarian failure," Journal of Biochemical and Molecular Toxicology, vol. 34, no. 12, Article ID e22591, 2020.

[41] G. Livera, R. Uzbekov, P. Jarrier et al., "Loss of oocytes due to conditional ablation of Murine double minute 2(Mdm2) gene is p53-dependent and results in female sterility," FEBS Letters, vol. 590, no. 16, pp. 2566-2574, 2016.

[42] S. Wang, S. Lin, M. Zhu et al., "Acupuncture reduces apoptosis of granulosa cells in rats with premature ovarian failure via restoring the PI3K/Akt signaling pathway," International Journal of Molecular Sciences, vol. 20, p. 24, 2019.

[43] T. A. Van Kempen, J. Gorecka, A. D. Gonzalez, F. Soeda, T. A. Milner, and E. M. Waters, "Characterization of neural estrogen signaling and neurotrophic changes in the accelerated ovarian failure mouse model of menopause," Endocrinology, vol. 155, no. 9, pp. 3610-3623, 2014.

[44] U. L. McClurg, N. C. T. H. Chit, M. Azizyan et al., "Molecular mechanism of the TP53-MDM2-AR-AKT signalling network regulation by USP12," Oncogene, vol. 37, no. 34, pp. 4679-4691, 2018.

[45] M. Qiu, J. Liu, Y. Su, R. Guo, B. Zhao, and J. Liu, "Diosmetin induces apoptosis by downregulating AKT phosphorylation via P53 activation in human renal carcinoma ACHN cells," Protein and Peptide Letters, vol. 27, no. 10, pp. 1022-1028, 2020.

[46] X. Deng, Y. Li, S. Gu et al., "p53 affects PGC1 $\alpha$ stability through AKT/GSK-3 $\beta$ to enhance cisplatin sensitivity in nonsmall cell lung cancer," Frontiers in Oncology, vol. 10, p. 1252, 2020.

[47] Y. Ogino, S. Tohyama, S. Kohno et al., "Functional distinctions associated with the diversity of sex steroid hormone receptors ESR and AR," The Journal of Steroid Biochemistry and Molecular Biology, vol. 184, pp. 38-46, 2018.

[48] H.-S. Lee, D.-W. Jung, S. Han et al., "Veterinary drug, 17 $\beta$ trenbolone promotes the proliferation of human prostate cancer cell line through the Akt/AR signaling pathway," Chemosphere, vol. 198, pp. 364-369, 2018.

[49] T. H. Pham, Y. L. Page, F. Percevault, F. Ferrière, G. Flouriot, and F. Pakdel, "Apigenin, a partial antagonist of the estrogen receptor (ER), inhibits ER-positive breast cancer cell proliferation through akt/FOXM1 signaling," International Journal of Molecular Sciences, vol. 22, no. 1, 2021.

[50] N. Abe, J. Watanabe, S. Tsunoda, H. Kuramoto, and I. Okayasu, "Significance of nuclear p-Akt in endometrial carcinogenesis: rapid translocation of p-Akt into the nucleus by estrogen, possibly resulting in inhibition of apoptosis," International Journal of Gynecological Cancer, vol. 21, no. 2, pp. 194-202, 2011.

[51] G. Gong, B. Qi, Y. T. Liang et al., "Danggui Buxue Tang, an ancient Chinese herbal decoction, protects $\beta$-amyloid-induced cell death in cultured cortical neurons," BMC Complementary and Alternative Medicine, vol. 19, no. 1, p. 9, 2019.

[52] M. Elkady, S. Shalaby, F. Fathi, and S. El-Mandouh, "Effects of quercetin and rosuvastatin each alone or in combination on cyclophosphamide-induced premature ovarian failure in female albino mice," Human \& Experimental Toxicology, vol. 38, no. 11, pp. 1283-1295, 2019.

[53] S. Roy, S. Banerjee, and T. Chakraborty, "Vanadium quercetin complex attenuates mammary cancer by regulating the P53, Akt/mTOR pathway and downregulates cellular proliferation correlated with increased apoptotic events," Biometals, vol. 31, no. 4, pp. 647-671, 2018.

[54] H. Luo, G. O. Rankin, Z. Li, L. Depriest, and Y. C. Chen, "Kaempferol induces apoptosis in ovarian cancer cells through activating p53 in the intrinsic pathway," Food Chemistry, vol. 128, no. 2, pp. 513-519, 2011.

[55] A. F. El-Kott, A. A. Shati, M. A. Al-Kahtani, and S. A. Alharbi, "Kaempferol induces cell death in A2780 ovarian cancer cells and increases their sensitivity to cisplatin by activation of cytotoxic endoplasmic reticulum-mediated autophagy and inhibition of protein kinase B," Folia Biologica, vol. 66, no. 1, pp. 36-46, 2020.

[56] X. Lv, Z. Xu, G. Xu et al., "Investigation of the active components and mechanisms of Schisandra chinensis in the treatment of asthma based on a network pharmacology approach and experimental validation," Food \& Function, vol. 11, no. 4, pp. 3032-3042, 2020. 